\title{
Definição de grupos genéticos aditivos visando melhor predição de valores genéticos em bovinos de corte
}

\author{
Prediction of breeding values in beef cattle using different definitions of additive \\ genetic groups
}

\author{
OLIVEIRA JÚNIOR, Gerson Antônio de ${ }^{1 *}$; ELER, Joanir Pereira ${ }^{1}$; FERRAZ, José \\ Bento Sterman ${ }^{1}$; PETRINI, Juliana ${ }^{2}$; MATTOS, Elisângela Chicaroni de ${ }^{1}$; MOURÃO, \\ Gerson Barreto ${ }^{2}$
}

\footnotetext{
${ }^{1}$ Universidade de São Paulo, Faculdade de Zootecnia e Engenharia de Alimentos, Departamento de Ciências Básicas, Pirassununga, São Paulo, Brasil.

${ }^{2}$ Universidade de São Paulo, Escola Superior de Agricultura "Luiz de Queiroz", Departamento de Zootecnia, Piracicaba, São Paulo, Brasil.

*Endereço para correspondência: gjunior@usp.br
}

\section{RESUMO}

O objetivo do estudo foi avaliar a estrutura de grupos genéticos aditivos como uma alternativa para melhor ajuste de animais com paternidade desconhecida nas avaliações genéticas. As características estudadas foram: peso a desmama; peso ao sobreano; ganho de peso pósdesmama em 345 dias; perímetro escrotal ao sobreano e o escore visual de musculosidade ao sobreano. Um banco em que todos os animais tinham paternidade conhecida foi utilizado como grupo controle. A partir deste, três cenários foram criados em que $30 ; 50$ ou $70 \%$ dos indivíduos foram aleatoriamente assumidos como animais com paternidade desconhecida. As estratégias consideradas de grupos genéticos aditivos foram: ano de nascimento do animal com paternidade desconhecida; fazenda de nascimento do animal com paternidade desconhecida; ano de nascimento e fazenda de nascimento do animal e um grupo controle negativo que manteve os animais com pais desconhecidos. A estratégia adequada de grupo genético aditivo foi escolhida como sendo aquela que resultou em um maior coeficiente de regressão e com valor genético aditivo mais próximos ao predito para os animais do grupo controle. Os resultados que incluíram a estratégia de grupo genético aditivo nos modelos de predição dos valores genéticos mostraram maior coerência com o grupo controle, frente a não inclusão destes nas análises. Dentre as estratégias propostas, ano de nascimento e fazenda de nascimento do animal e apenas ano de nascimento do animal com paternidade desconhecida obtiveram os melhores resultados.

Palavras-chave: correlação, monta natural, pecuária, regressão linear, reprodutor múltiplo

\section{SUMMARY}

The paper aims to analyze the structure of additive genetic groups as an alternative to include animals with unknown parentage in genetic evaluation. Several traits were studied: weaning weight; yearling weight; post-weaning weight gain in 345 days; scrotal circumference at 18 months of age; and visual muscling scores at 18 months of age. As a control group it was used a database where all animals had known paternity. Thus, three scenarios were defined where $30 \% ; 50 \%$ or $70 \%$ of the observations were randomly taken as animals with unknown parentage. For these three simulated databases, the strategies considered to define additive genetic groups were: effect of year at birth; effect of farm at birth; effect of year and farm at birth; and a negative control group, which considered animals with unknown paternity. The most appropriate strategy for additive genetic group was chosen as the one that resulted in a higher regression coefficient and additive genetic value closest to the predicted for those animals in the control group. The results considering the strategy of additive genetic show large agreement with the control group. Among the strategies proposed, farm of 
birth and year of birth of the animal with unknown parentage achieved the best results.

Keywords: correlation, livestock, linear regression, multiple sire, natural mating

\section{INTRODUÇÃO}

A bovinocultura de corte brasileira destaca-se pela produção extensiva de animais, especialmente da raça Nelore, os quais são mantidos em regime de pastagens (MILLEN et al., 2011). Neste sistema, principalmente em grandes rebanhos comerciais, prevalece o uso da monta natural. Este manejo representa cerca de $90 \%$ dos acasalamentos entre bovinos de corte no Brasil (ASBIA, 2011).

No manejo de monta natural é comum a utilização de reprodutores múltiplos (RM), em que um grupo de reprodutores é utilizado no acasalamento com um lote de fêmeas. Um problema associado a esse manejo é que a paternidade dos bezerros nascidos não pode ser determinada, a menos que se utilize a técnica de genotipagem (HEYEN et al., 2009). Embora esta seja a técnica mais confiável de reconhecimento de paternidade, ela exige um alto investimento financeiro quando utilizada em grande escala, o que tem inviabilizado seu uso em grandes rebanhos (SAPP et al., 2007; GOMEZ-RAYA et al., 2008).

Os grupos genéticos aditivos foram inicialmente desenvolvidos como estratégia para os animais fundadores da população e representam efeitos referentes ao parentesco não contabilizados na análise (PHOCAS \& LALOË, 2004). Esses grupos são, portanto, atribuídos apenas aos animais cujas informações de parentesco são incompletas. A informação de grupo genético é incluída nas equações de modelo misto, pelo modelo animal
(HENDERSON, 1977) e utilizada como substituta do parental nas análises para predição do mérito genético desses animais. Desta forma, o efeito do grupo genético representa a contribuição genética média que o critério escolhido para suprir a informação de pai ausente teria sobre as progênies que possuem registros de produção (WESTELL et al., 1988). Ou seja, o mérito genético predito do animal seria um valor corrigido em função do grupo genético a que este pertenceria (THERON et al., 2003).

Visto a não existência de um consenso em como tratar o problema de paternidade desconhecida, é indispensável o foco em estudos que analisem metodologias de formação de grupos genéticos referentes à predição de valores genéticos, de modo que se determine a estrutura de grupo adequada às avaliações dos rebanhos em programas de melhoramento genético animal.

Dessa forma, objetivou-se estudar a formação de grupos genéticos como abordagem para a inclusão de animais com paternidade desconhecida nas avaliações genéticas, analisando-a quanto aos seus efeitos sobre a predição dos valores genéticos.

\section{MATERIAL E MÉTODOS}

$\mathrm{O}$ experimento foi conduzido junto ao Grupo de Melhoramento Genético Animal e Biotecnologia (GMAB) da Faculdade de Zootecnia e Engenharia de Alimentos da Universidade de São Paulo (FZEA/USP), localizado em Pirassununga, São Paulo. Os dados analisados pertencem ao Programa de Melhoramento Genético da Agropecuária CFM, que é desenvolvido junto ao grupo. As informações são 
referentes a animais da raça Nelore, nascidos entre os anos de 1984 e 2009, em quinze fazendas localizadas nos Estados de São Paulo, Mato Grosso do Sul e Goiás.

O banco de dados contabilizou informações de 116.368 indivíduos com registros de produção, resultando uma matriz de parentesco com 175.398 animais, sendo 1.769 diferentes touros e 80.928 vacas. Os animais foram mantidos em pastagens de alta qualidade $(40 \%$ Brachiaria brizanta, $50 \%$ Panicum maximum e $10 \%$ outras) recebendo apenas sal mineral como suplementação até os dezoito meses de idade.

As informações existentes no banco de dados eram: a identificação dos animais, dos pais, grupos de contemporâneos, fazenda de nascimento, ano de nascimento, peso a desmama (PESDES, $\mathrm{kg}$ ); peso ao sobreano mensurado próximo de 18 meses de idade (PSOB, $\mathrm{kg}$ ); ganho de peso pós-desmama em 345 dias (GPSOB, $\mathrm{kg} / \mathrm{dia}$ ), correspondendo ao ganho da desmama aos 18 meses de idade; perímetro escrotal ao sobreano (PE18, cm) e escore visual de musculosidade ao sobreano (MUSC18, pontos), mensurados próximos aos 18 meses de idade; além das idades relacionadas a cada pesagem ou mensuração e a idade da mãe ao parto (IMP).

Os grupos de contemporâneos foram formados pela concatenação das informações de fazenda, ano de nascimento e sexo do animal e lote nas respectivas fases de mensuração. O lote dentro de fazenda e safra representa o grupo de manejo.

As informações utilizadas foram previamente editadas para minimizar inconsistência dos resultados. Foram desconsideradas as informações dos animais com pais e/ou mães desconhecidos; animais produtos de transferência de embrião; com informações fora da amplitude de valores biologicamente aceitáveis; pertencentes aos grupos de contemporâneos com menos de vinte animais com mensuração dentro do intervalo proposto; pertencentes a grupos de contemporâneos sem variabilidade nos dados de produção; filhos de touros com apenas uma progênie; sem nenhum dado de produção mensurado; com idade da mãe ao parto desconhecida (Tabela 1).

Tabela 1. Estatísticas descritivas das características estudadas e o número de animais com paternidade desconhecida após sorteio de 30 (GG30), 50 (GG50) e 70\% (GG70) dos indivíduos do banco de dados

\begin{tabular}{lccccc}
\hline Item & PESDES & PESO18 & GPSOB & PE18 & MUSC18 \\
\hline $\mathrm{N}$ & 108.380 & 81.675 & 80.152 & 40.559 & 79.148 \\
Média & 185,60 & 308,40 & 115,60 & 27,60 & 3,70 \\
MIN & 109,0 & 186,0 & 0,1 & 17,5 & 1,0 \\
MAX & 261,0 & 429,0 & 312,6 & 37,5 & 6,0 \\
DP & 26,77 & 43,11 & 31,32 & 3,22 & 0,94 \\
GG30 & 32.514 & 24.046 & 24.503 & 23.744 & 12.1689 \\
GG50 & 54.190 & 40.121 & 40.848 & 39.574 & 20.280 \\
GG70 & 75.866 & 56.170 & 57.185 & 55.404 & 28.392 \\
\hline
\end{tabular}

PESDES = peso a desmama (kg); PESO18 = peso aos dezoito meses de idade $(\mathrm{kg}) ;$ GPSOB $=$ ganho de peso pós-desmama em 345 dias ( $\mathrm{kg} / \mathrm{dia}) ;$ PE18 = perímetro escrotal aos dezoito meses de idade $(\mathrm{cm})$; MUSC18 = escore de musculosidade aos dezoito meses de idade (pontos); $\mathrm{N}=$ número de animais; MIN = valor mínimo encontrado para a característica; $\mathrm{MAX}=$ valor máximo encontrado para a característica; $\mathrm{DP}=$ desvio padrão. 
Ao final da preparação do banco de dados, todos os animais apresentavam genealogia completa. A partir disso, três cenários foram criados, baseados em análises prévias, em que $30 \%, 50 \%$ ou $70 \%$ dos indivíduos foram aleatoriamente assumidos como animais com paternidade desconhecida (Tabela 1). Para tal procedimento, utilizou-se $\mathrm{o}$ PROC SURVEYSELECT do programa estatístico SAS (SAS Institute, 2008), trabalhando com a metodologia de amostragem simples, que proporciona a cada animal a mesma probabilidade de ser escolhido.

Quatro estratégias de estrutura dos grupos genéticos foram analisadas, sendo ano de nascimento do animal com paternidade desconhecida (SAFRA); fazenda de nascimento do animal com paternidade desconhecida (FAZNAS); ano de nascimento e fazenda de nascimento do animal (FAZSAFRA) e um grupo controle negativo, que considerou os animais com pais desconhecidos (ZERO).

As análises genéticas realizadas foram unicaracterística. $\mathrm{O}$ modelo estatístico que considerou apenas os animais com parentesco conhecido, foi denominado de "modelo controle". As equações de modelo misto para esses animais foram:

$\mathrm{y}=\mathrm{X} \beta+\mathrm{Za}+\mathrm{Wm}+\mathrm{Sc}+\mathrm{Td}+\varepsilon$

$y=X \beta+Z a+W m+S c+T d+Q g+\varepsilon(2)$, em que $\mathbf{y}$ é o vetor das variável dependente (GPSOB, por exemplo); $\boldsymbol{\beta}$ é $o$ vetor dos efeitos fixos, incluindo as covariáveis; $\mathbf{X}$ é a matriz de incidência, associando os elementos de $\boldsymbol{\beta}$ a $\mathbf{y}$; a é o vetor dos valores genéticos aditivos direto; $\mathbf{m}$ é o vetor dos valores genéticos aditivos materno; c é o vetor dos efeitos aleatórios não correlacionados de ambiente permanente materno; d é o vetor dos efeitos aleatórios não correlacionados de grupo de manejo a desmama; $\boldsymbol{g}$ é o vetor de efeitos fixos referentes aos grupos genéticos aditivos; $\mathbf{Z}, \mathbf{W}, \mathbf{S}, \mathbf{T}$ e $\mathbf{Q}$ representam as matrizes de incidência dos efeitos aleatórios, associando respectivamente os elementos de $\mathbf{a}, \mathbf{m}, \boldsymbol{c}, \mathbf{d}$ e $\boldsymbol{g}$ à variável dependente $\mathbf{y} ; \boldsymbol{\varepsilon}$ o vetor do efeito residual inerente a cada observação. As pressuposições assumidas foram:

$$
E\lceil y\rceil=X \beta \quad E\left[\begin{array}{l}
\mathbf{a} \\
\mathbf{m} \\
\mathbf{c} \\
\mathbf{d} \\
\mathbf{\varepsilon}
\end{array}\right]=\left[\begin{array}{l}
\mathbf{0} \\
\mathbf{0} \\
\mathbf{0} \\
\mathbf{0} \\
\mathbf{0}
\end{array}\right] \quad \boldsymbol{V a r}\left[\begin{array}{l}
\mathbf{a} \\
\mathbf{m} \\
\mathbf{c} \\
\mathbf{d} \\
\mathbf{\varepsilon}
\end{array}\right]=\left[\begin{array}{ccccc}
\mathbf{A} \sigma_{\mathrm{a}}^{2} & 0 & 0 & 0 & 0 \\
0 & \mathbf{A} \sigma_{m}^{2} & 0 & 0 & 0 \\
0 & 0 & \mathbf{I} \sigma_{c}^{2} & 0 & 0 \\
0 & 0 & 0 & \mathbf{I} \sigma_{d}^{2} & 0 \\
0 & 0 & 0 & 0 & \mathbf{I} \sigma_{z}^{2}
\end{array}\right],
$$

em que $\boldsymbol{\sigma}_{a}^{2}$ representa o componente de variância genética aditiva; $\boldsymbol{\sigma}_{m}^{2} \quad \mathrm{o}$ componente de variância genética materna; $\boldsymbol{\sigma}_{c}^{2}$ o componente de variância de ambiente permanente materno; $\boldsymbol{\sigma}_{d}^{2} \mathrm{o}$ componente de variância do efeito aleatório de grupo de manejo a desmama (GMANDES); $\boldsymbol{\sigma}_{e}^{2}$ é o componente de variância residual; $\boldsymbol{A}$ representa a matriz de parentesco entre todos os animais participantes do banco e $\boldsymbol{I}$ uma matriz identidade.

O efeito de GMANDES foi separado do grupo de contemporâneos (GC) e tratado como efeito aleatório não correlacionado nos modelos das características pós-desmama. Como descrito por Santana Júnior et al. (2011), esta abordagem proporciona uma menor subdivisão dos $\mathrm{GC}$ e, consequentemente, um significativo aumento do número de animais por $\mathrm{GC}$ 
e também de animais mantidos no banco de dados.

As estimativas de componentes de variância foram obtidas por máxima verossimilhança restrita utilizando $\mathrm{o}$ pacote computacional VCE-6 (KOVAC \& GROENEVELD, 2007). Com base nesses $\mathrm{CV}$, os valores genéticos aditivos foram preditos pelo modelo animal, para todos os animais da matriz de parentesco, com a utilização do programa PEST (GROENEVELD, 2006) utilizando os mesmos modelos da estimação de componentes de variância. Nos trabalhos de (CARDOSO \& TEMPELMAN, 2004; SHIOTSUKI et al., 2012), os autores não relataram diferenças significativas na predição dos componentes de variância. Com isso, os componentes estimados para o banco controle (modelo 1) foram utilizados nas análises com grupos genéticos (modelo 2).

Os valores genéticos preditos para os animais pertencentes aos bancos de dados com animais sem informação paterna foram contrastados com os valores genéticos preditos para os animais com genealogia completa, a partir de uma análise de regressão linear feita pelo procedimento PROC REG do software SAS 9.2 (SAS Institute, 2008). A equação pode ser descrita como:

$y=\mu+\beta x+e$,

em que $y$ é o valor genético predito pelo banco com GG; $\mu$ é a média dos valores genéticos para o bancol; $\beta$ é o coeficiente angular da reta que relaciona $x$ a $y$; e é o resíduo inerente a cada observação.

Para visualizar o real impacto gerado pela ausência da informação paterna, os valores genéticos aditivos dos animais que tiveram a informação de pai excluída foram comparados com os mesmos animais preservando a paternidade. O ranking dos valores genéticos preditos antes e depois da modificação da identidade de pai desses animais foi comparado pela correlação de Spearman.

\section{RESULTADOS E DISCUSSÃO}

Os coeficientes de herdabilidade para as características estudadas corroboram os valores encontrados na literatura (BOLIGON et al. 2010; MELIS et al., 2010; SANTANA et al., 2011) conforme apresentado na Tabela 2. Tais resultados validam os componentes de variância estimados no trabalho e indicam o nível de variabilidade fenotípica das características, ressaltando a possibilidade de utilizá-las como critério de seleção em animais da raça Nelore.

O componente de variância referente ao efeito de grupo de manejo ao desmame (GMANDES), dado como proporção da variância fenotípica, apresentou valores importantes para as características PESO18 e GP345. Não houve efeito importante nas demais características, sendo o menor valor 0,03 PE18.

Os resultados dos coeficientes de regressão linear mostraram que os valores genéticos preditos pelo modelo alternativo (modelo 2) foram inferiores aos do grupo controle (modelo 1, Tabela 3). Tal fato era esperado, visto que as estratégias de grupos genéticos não substituem a informação paterna, apenas contornam o problema, sendo utilizadas somente quando informações do pedigree são desconhecidas (WESTELL et al., 1988).

Como era esperado, o aumento da proporção de pais desconhecidos resultou em maiores diferenças entre as predições dos valores genéticos. Os resultados corroboram Pollak \& Quaas (1983), que afirmaram que a necessidade dos GG era inversamente proporcional ao número de informações no pedigree. 
Tabela 2. Componente de variância e coeficiente de herdabilidade para peso a desmama (PESDES), peso ao sobreano (PESO18), ganho de peso pós-desmame em 345 dias (GP345), perímetro escrotal aos dezoito meses de idade (PE18) e escore visual de musculosidade aos dezoito meses de idade

\begin{tabular}{lrccccccc}
\hline Característica & \multicolumn{1}{c}{$\sigma_{a}^{2}$} & $\sigma_{m}^{2}$ & $\sigma_{c}^{2}$ & $\sigma_{d}^{2}$ & $\sigma_{a}^{2}$ & $\sigma_{p}^{2}$ & $h_{a}^{2}$ & $h_{m}^{2}$ \\
\hline PESDES (kg) & 83,20 & 24,21 & 0,16 & -- & 167,41 & 327,07 & 0,25 & 0,07 \\
PESO18 (kg) & 237,84 & -- & -- & 0,07 & 359,96 & 645,38 & 0,37 & -- \\
GP345 (kg) & 78,37 & -- & -- & 0,15 & 257,35 & 397,05 & 0,20 & -- \\
PE18 (cm) & 3,41 & -- & -- & 0,03 & 3,40 & 7,04 & 0,48 & - \\
MUSC18 (uni.) & 0,14 & -- & -- & 0,04 & 0,48 & 0,64 & 0,21 & -- \\
\hline
\end{tabular}

$\sigma_{a}^{2}=$ variância genética aditiva; $\sigma_{m}^{2}=$ variância genética aditiva materna; $\sigma_{\theta}^{2}=$ variância dos efeitos de ambiente da vaca, dada como proporção da variância fenotípica; $\sigma_{d}^{2}=$ variância dos efeitos de GMANDES, dada como proporção da variância fenotípica; $\sigma_{e}^{2}=$ variância residual; $\sigma_{p}^{2}=$ variância fenotípica; $h_{a}^{2}=$ coeficiente de herdabilidade do efeito genético direto; $h_{m}^{2}=$ coeficiente de herdabilidade do efeito genético materno.

Tabela 3. Valor médio (desvio padrão) relacionado ao coeficiente de regressão linear simples dos valores genéticos preditos pelo modelo [2] considerando 30, 50 ou $70 \%$ dos animais com paternidade desconhecida, em função dos valores obtidos pelo modelo com pais conhecidos [1], para as características de peso a desmama (PESDES, kg), peso aos dezoito meses de idade (PESO18, Kg), ganho de peso pós-desmama em 345 dias (GPSOB, Kg/dia), perímetro escrotal aos dezoito meses de idade (PE18, cm) e escore de musculosidade aos dezoito meses de idade (MUSC18, pontos)

\begin{tabular}{llccccc}
\hline Cenário (\%) & Estratégia & PESDES & PESO18 & GP345 & PE18 & MUSC18 \\
\hline \multirow{4}{*}{30} & FAZNAS & $0,81(0,007)$ & $0,85(0,006)$ & $0,79(0,009)$ & $0,93(0,005)$ & $0,81(0,007)$ \\
& FAZSAFRA & $0,93(0,007)$ & $1,02(0,005)$ & $0,95(0,009)$ & $1,01(0,005)$ & $0,94(0,009)$ \\
& SAFRA & $0,92(0,007)$ & $1,01(0,006)$ & $0,94(0,008)$ & $1,00(0,003)$ & $0,93(0,009)$ \\
& ZERO & $0,69(0,005)$ & $0,69(0,005)$ & $0,67(0,007)$ & $0,85(0,004)$ & $0,72(0,006)$ \\
\hline \multirow{4}{*}{50} & FAZNAS & $0,75(0,008)$ & $0,80(0,006)$ & $0,71(0,010)$ & $0,90(0,005)$ & $0,72(0,007)$ \\
& FAZSAFRA & $0,88(0,010)$ & $1,00(0,006)$ & $0,89(0,010)$ & $0,99(0,006)$ & $0,87(0,011)$ \\
& SAFRA & $0,87(0,010)$ & $0,99(0,007)$ & $0,88(0,012)$ & $0,98(0,007)$ & $0,85(0,010)$ \\
& ZERO & $0,64(0,004)$ & $0,64(0,004)$ & $0,59(0,006)$ & $0,81(0,003)$ & $0,64(0,007)$ \\
\hline \multirow{4}{*}{70} & FAZNAS & $0,70(0,009)$ & $0,77(0,007)$ & $0,65(0,008)$ & $0,88(0,005)$ & $0,66(0,007)$ \\
& FAZSAFRA & $0,84(0,012)$ & $0,98(0,010)$ & $0,83(0,011)$ & $0,98(0,007)$ & $0,83(0,015)$ \\
& SAFRA & $0,83(0,012)$ & $0,97(0,008)$ & $0,82(0,015)$ & $0,97(0,008)$ & $0,81(0,015)$ \\
& ZERO & $0,60(0,003)$ & $0,61(0,003)$ & $0,54(0,004)$ & $0,79(0,003)$ & $0,58(0,005)$ \\
\hline
\end{tabular}

FAZNAS = fazenda de nascimento do animal; FAZSAFRA = fazenda e safra de nascimento do animal; SAFRA = ano de nascimento do animal; ZERO = ausência de grupo genético.

Os desvios-padrão estimados foram pequenos, sugerindo baixa variabilidade dos valores médios. As estratégias de agrupamento de FAZSAFRA e SAFRA obtiveram coeficientes de regressão mais próximos de um, em todas as 
características estudadas. Esses resultados sugerem que estas estratégias predizem de forma menos viesada os valores genéticos dos animais com paternidade ausente. Quando os grupos genéticos foram desconsiderados (ZERO), os coeficientes foram ainda menores, indicando maiores prejuízos na predição dos valores genéticos.

O resultado médio para os animais com pedigree completo foi bem superior aos demais (Tabela 4). Entretanto, todas as correlações de rankings foram altas, sugerindo que os resultados apresentem certa tendência de variação, não havendo grandes alterações na escolha dos melhores animais para reprodutores. Nos resultados para a característica de peso a desmama (PESDES) no cenário de $30 \%$ de pais desconhecidos, a análise que não considerou a metodologia dos grupos (ZERO) proporcionou resultados ligeiramente mais coerentes com os considerados "verdadeiros" (modelo 1), quando comparados com a estratégia FAZNAS, obtendo coeficientes de correlações de 0,85 e 0,83 , respectivamente. Para todas as outras análises realizadas, a não inclusão do efeito fixo de grupos genéticos aditivos (ZERO) resultou em menores médias dos valores genéticos.

Tabela 4. Valor genético aditivo médio e correlação de Spearman entre os animais que perderam informação de pai $(30,50$ e $70 \%)$ e o grupo controle, para as características de peso a desmama (PESDES, Kg), peso aos dezoito meses de idade (PESO18, Kg), ganho de peso pós-desmama em 345 dias (GPSOB, $\mathrm{Kg} / \mathrm{dia}$ ), perímetro escrotal aos dezoito meses de idade $(\mathrm{PE} 18, \mathrm{~cm})$ e escore de musculosidade aos dezoito meses de idade (MUSC18, pontos)

\begin{tabular}{clccccc}
\hline \multirow{2}{*}{ Cenário (\%) } & Estratégia & PESDES & PESO18 & GP345 & PE18 & MUSC18 \\
\cline { 2 - 7 } & Controle & 6,45 & 9,85 & 3,49 & 0,88 & 0,16 \\
\hline \multirow{2}{*}{30} & FAZNAS & 1,$32 ; 0,83$ & 2,$12 ; 0,95$ & 0,$91 ; 0,92$ & 0,$22 ; 0,94$ & 0,$04 ; 0,92$ \\
& FAZSAFRA & 1,$19 ; 0,89$ & 2,$40 ; 0,98$ & 0,$90 ; 0,89$ & 0,$23 ; 0,95$ & 0,$05 ; 0,95$ \\
& SAFRA & 1,$30 ; 0,96$ & 2,$32 ; 0,98$ & 1,$02 ; 0,89$ & 0,$27 ; 0,95$ & 0,$04 ; 0,95$ \\
& ZERO & 0,$72 ; 0,85$ & 1,$00 ; 0,78$ & 0,$25 ; 0,71$ & 0,$11 ; 0,89$ & 0,$03 ; 0,87$ \\
\hline \multirow{2}{*}{50} & FAZNAS & 1,$32 ; 0,85$ & 2,$07 ; 0,92$ & 0,$67 ; 0,78$ & 0,$23 ; 0,96$ & 0,$03 ; 0,85$ \\
& FAZSAFRA & 1,$24 ; 0,93$ & 2,$51 ; 0,97$ & 0,$98 ; 0,93$ & 0,$24 ; 0,95$ & 0,$04 ; 0,92$ \\
& SAFRA & 1,$33 ; 0,93$ & 2,$35 ; 0,97$ & 0,$80 ; 0,89$ & 0,$27 ; 0,95$ & 0,$04 ; 0,92$ \\
& ZERO & 0,$56 ; 0,85$ & 0,$81 ; 0,75$ & 0,$15 ; 0,68$ & 0,$08 ; 0,87$ & 0,$02 ; 0,79$ \\
\hline \multirow{2}{*}{70} & FAZNAS & 1,$25 ; 0,80$ & 2,$10 ; 0,89$ & 0,$65 ; 0,77$ & 0,$23 ; 0,92$ & 0,$03 ; 0,80$ \\
& FAZSAFRA & 1,$42 ; 0,88$ & 2,$46 ; 0,96$ & 0,$90 ; 0,87$ & 0,$23 ; 0,94$ & 0,$04 ; 0,88$ \\
& SAFRA & 1,$42 ; 0,90$ & 2,$40 ; 0,96$ & 0,$79 ; 0,88$ & 0,$23 ; 0,94$ & 0,$04 ; 0,88$ \\
& ZERO & 0,$48 ; 0,75$ & 0,$79 ; 74$ & 0,$19 ; 0,66$ & 0,$08 ; 0,86$ & 0,$01 ; 0,73$ \\
\hline
\end{tabular}

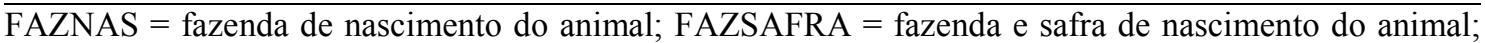
SAFRA = ano de nascimento do animal; ZERO = ausência de grupo genético.

As perdas de informações de pedigree é comum em propriedade que trabalham com pecuária extensiva, onde este tipo de informação pode ser difícil de obter
(CASELLAS et al., 2006). Os atores, assim como Phocas \& Laloë (2004), discutem que a aquisição de animais com pouca ou nenhuma informação de 
pedigree é uma prática comum entre os produtores, e que estes animais podem prejudicar o progresso genético da população por não terem valores genéticos devidamente preditos. No trabalho de Phocas \& Laloë (2004), quando o efeito de grupo genético foi desprezado dos modelos estatísticos, os valores genéticos preditos tendiam a zero. Tais resultados coincidem com os obtidos para as análises que desconsideraram os GG (ZERO), com médias tendendo a zero à medida que aumentam o número de animais sem informação paterna. Tais resultados corroboram os mostrados pelos coeficientes de regressão linear (Tabela 2 ), em que os valores genéticos preditos para os animais sem informação paterna (ZERO) foram inferiores aos preditos na presença dos grupos genéticos. A não inclusão dos grupos genéticos nos modelos também resultou em menores valores de correlações entre os rankings dos animais para todas as características estudadas.

As estratégias FAZSAFRA e SAFRA obtiveram os mesmos resultados de correlação nas características de peso ao sobreano (PSOB), perímetro escrotal aos sobreano (PE18) e escore de musculosidade ao sobreano (MUSC18), independentemente da proporção de pais desconhecidos. Em todos os casos, estas proporcionaram melhores resultados que a estratégias FAZNAS.

Em peso a desmama e ganho de peso pósdesmama as estratégias FAZSAFRA e SAFRA também apresentaram maiores coeficientes de correlação, com resultados próximos entre elas.

Conclui-se a que o uso de grupos genéticos aditivos com a finalidade de corrigir a ausência da informação paterna é uma estratégia válida. Os resultados mostraram maior coerência com o grupo controle (modelo 1) quando os valores genéticos foram preditos com o uso da metodologia, frente a não inclusão destes nas análises genéticas. Apesar dos valores genéticos menores, essa diferença não alterou de forma significativa os rankings dos animais. Assim, o uso de grupos genéticos aditivos permite que os melhores reprodutores sejam selecionados mesmo sem a informação de genealogia, favorecendo o progresso genético da população.

Os resultados mostraram, ainda, que as melhores estratégias entre as que foram propostas foram FAZSAFRA e SAFRA, com resultados mais próximos aos obtidos pelo grupo controle.

\section{AGRADECIMENTOS}

À agência de fomento CAPES e ao Grupo de Melhoramento Animal e Biotecnologia (GMAB) por ter cedido o banco de dados.

\section{REFERÊNCIAS}

\author{
ASSOCIAÇÃO BRASILEIRA DE \\ INSEMINAÇÃO ARTIFICIAL - \\ ASBIA. Relatório estatístico de \\ produção, importação e \\ comercialização de sêmen., 2011. \\ Disponível em: \\ $<$ http://www.asbia.org.br/novo/upload/ \\ mercado/relatorio2011.pdf.> Acesso \\ em: 15 nov. 2012.
}

BERTAZZO, R. P.; FREITAS, R.T.F.de; GONÇALVES, T. de M.; PEREIRA, I.G.;ELER, J.P.;FERRAZ, J.B.S.; OLIVEIRA, A.I.G. de; ANDRADE, I.F. de. Parâmetros genéticos de longevidade e produtividade de fêmeas da raça Nelore. Revista Brasileira de Zootecnia, v.33, n.5, p.1118-1127, 2004. 
BOLIGON, A.A.; ALBUQUERQUE, L.G. de; MERCADANTE, M.E.Z.;

LÔBO, R.B. Study of relations among age at first calving, average weight gains and weights from weaning to maturity in Nellore cattle. Revista Brasileira de Zootecnia, v.39, n.4, p.746-751, 2010.

CARDOSO, F.F.; TEMPELMAN, R.J. Genetic evaluation of beef cattle accounting for uncertain paternity. Livestock Production Science, v.89, p.109-120, 2004.

CASELLAS, J.; PIEDRAFITA, J.; VARONA, L. Bayes factor for testing between different structures of random genetic groups: A case study using weaning weight in Bruna dels Pirineus beef cattle. Genetics Selection

Evolution, v.39, p.39-53, 2006.

FORNI, S.; FEDERICI, J.F.; ALBUQUERQUE, L.G. Tendências genéticas para escores visuais de conformação, precocidade e musculatura à desmama de bovinos Nelore. Revista

Brasileira de Zootecnia, v.36, n.3, p.572-577, 2007.

GOMEZ-RAYA, L.; PRIEST, K.; RAUW, W.M.; OKOMO-ADHIAMBO, M.; THAIN, D.; BRUCE, B.; RINK, A.; TORELL, R.; GRELLMAN, L.; NARAYANAN, R.; BEATTIE, C.W. The value of DNA paternity identification in beef cattle: examples from Nevada's free-range ranches. Journal of Animal Science, v.86, p.17-24, 2008.

GROENEVELD, E. PEST, user's manual. Germany: Institute of Animal Science, 2006.

HENDERSON, C.R. Best linear unbiased prediction of breeding values not in the model for records. Journal of Dairy Science, v.60, n.5, p.783-787, 1977.
HEYEN, D.W.; BEEVER, J.E.; DA, Y.; EVERT, R.E.; GREEN, C.; BATES, S.R.E.; ZIEGLE, J.S.; LEWIN, H.A. Exclusion probabilities of 22 bovine microsatellite markers in fluorescent multiplexes for semiautomated parentage testing. Animal Genetics, v.28, n.1, p.21-27, 2009.

HORIMOTO, A.R.; FERRAZ, J.B.; BALIEIRO, J.C.; ELER, J.P.

Phenotypic and genetic correlations for body structure scores (frame) with productive traits and index for CEIP classification in Nellore beef cattle. Genetics and Molecular Research, v.6, n.1, p.188-196, 2007.

KOVAC, M.; GROENEVELD, E. VCE-6 user's guide and reference manual. Version 6. Slovenia: University of Ljubljana, 2007. 75p.

MELIS, M.H.V. et al. Additive genetic relationship of longevity with fertility and production traits in Nellore cattle based on bivariate models. Genetics and Molecular Research, v.9, n.1, p.176-187, 2010.

MILLEN, D.D.; PACHECO, R.D.L.; MEYER, P.M.; RODRIGUES, P.H.M.; ARRIGONI, M.D.B. Current outlook and future perspectives of beef production in Brazil. Animal Frontiers, v.1, n.2, p.46-52, 2011.

PHOCAS, F.; LALOË, D. Should genetic groups be fitted in BLUP evaluation? Practical answer for the French AI beef sire evaluation.

Genetics Selection Evolution, v.36, n.3, p.1-21, 2004.

POLLAK, E.J.; QUAAS, R.L. Definition of group effects in sire evaluation models. Journal of Dairy Science, v.66, p.1503-1509, 1983. 
REGATIERI, I.C.; BOLIGON, A.A.; ALBUQUERQUE, L.G. Genetic analysis of visual scores and their relationships to mature female weight in Nellore breed. Revista Brasileira de Zootecnia, v.40, n.1, p.100-105, 2011.

SAPP, R.L.; ZHANG, W.; BERTRAND J.K.; REKAYA, R. Genetic evaluation in the presence of uncertain additive relationships. I. Use of phenotypic information to ascertain paternity. Journal of Animal Science, v.85, n.10, p.2391-2400, 2007.

SANTANA JUNIOR, M.L.; ELER, J.P.; FERRAZ, J.B.S.; MATTOS, E.C. Genetic relationship between growth and reproductive traits in Nellore cattle. Animal, v.1, p.1-6, 2011.

SAS Institute. SAS User's guide:

Statistics. Version 9.2. Cary, NC., 2008.

SHIOTSUKI, L.; CARDOSO, F.F.; SILVA, J.A.I.V.; ROSA, G.J.M.; ALBUQUERQUE, L.G. Evaluation of an average numerator relationship matrix model and a Bayesian hierarchical model for growth traits in Nellore cattle with uncertain paternity. Livestock Science, v.144, p.89-95, 2012.
SHIOTSUKI, L.; SILVA, J.A.V.; TONHATI, H.; ALBUQUERQUE, L.G. Genetic associations of sexual precocity with growth traits and visual scores of conformation, finishing, and muscling in Nelore cattle. Journal of Animal Science, v.87, n.5, p.15911597, 2009.

THERON, H.E.; KANFER, F.H.J.; RAUTENBACH, L. The effect of phantom parent groups on genetic trend estimation. South African Journal of Animal Science, v.32, p.130-135, 2003.

TORAL, F.L.B.; SILVA, L.O.C. da; MARTINS, E.N.; GONDO, A.;

SIMONELLI, S.M. Interação genótipo× ambiente em características de crescimento de bovinos da raça Nelore no Mato Grosso do Sul. Revista

Brasileira de Zootecnia, v.33, n.6, p.1445-1455, 2004.

WESTELL, R.A.; QUAAS, R.L.; Van VLECK, L.D. Genetic groups in an animal model. Journal of Dairy Science, v.71, p.1310-1318, 1988.

Data de recebimento: 14/02/2013

Data de aprovação: 12/06/2013 